\title{
Myopia: Induced, Normal and Clinical
}

\author{
A L HOLDEN, W HODOS, B P HAYES and F W FITZKE \\ London
}

\begin{abstract}
Summary
A review is given of lid-suture myopia in monkeys, of the natural lower field myopia in the pigeon eye, and of myopias in chick eyes produced by visual occluders. The ametropias produced by ophthalmic lenses, and the pharmacology of experimental myopia, are reviewed. Human studies are reviewed which can be taken as models of the experimental myopias. Two theories of myopic growth are outlined and evaluated.
\end{abstract}

\section{Clinical Myopia}

There have been a number of excellent clinical reviews of myopia, by Duke-Elder, ${ }^{1,2}$ Blach, ${ }^{3}$ and recently the impressive book by Curtin. ${ }^{4}$ Duke-Elder wrote in 1949 that "The aetiology of myopia has excited an immense amount of speculation and controversy ever since ophthalmology has become a science, and the theories which have been put forward to explain its development are as ingenious, fanciful and contradictory as have accumulated around any subject in medicine." The purpose of the present paper is to review recent laboratory studies of myopic development in animal eyes, which have examined environmental factors producing myopic growth. These studies allow theories to be tested, and verified or falsified, and could in time move to a causal explanation of myopia, and to a rational treatment of its minor and morbid forms. Most studies have applied a procedure (for example lid-suture, an occluder, or pharmacological intervention), to one eye only. This permits the fellow eye to be used as a control which is genetically identical and which is subject to identical systemic effects.

It has long been the practice to distinguish between these forms as 'physiological' and 'pathological'. 1,2,3 Curtin $^{4}$ distinguishes between three varieties, 'physiologic, intermediate, and pathological'. All, however, describe a condition where the eye becomes short-sighted (clear vision is possible only for near objects, unless refractive corrections are used), and the eye is of increased axial length. The pathological myopias include a number of fundus signs, such as straightening of the retinal vessels, the myopic crescent, and in severe cases a staphyloma and tapetoretinal degeneration at the posterior pole. Simple myopia can be regarded as an error in growth of the eye, where axial length is too long for essentially normal optical components. Pathological myopia can be regarded as including excessive elongation of the eye because of inadequate maintenance of scleral strength and thickness.

\section{Lid-suture myopia in monkeys}

Wiesel and Raviola ${ }^{5}$ reported in 1977 that lidsuture in neonatal monkeys could produce myopic growth. After suturing the lids for periods up to 26 months, eyes were found to be substantially myopic by retinoscopy, ( -1 to $-13.5 \mathrm{D})$, and of increased axial length and equatorial diameter. The posterior sclera was thinner than in the fellow eye. The anterior segment of the eye was essentially similar on the two sides. Their description of this myopic model is thus parallel to the classical human case 
of Heine, ${ }^{6}$ reported in 1899. Although Wiesel and Raviola offered no explanation for their important result, they carried out two further experiments to test alternative theories.

Firstly, they reported, in 1978, that darkrearing animals with lid-suture resulted in normal refractions and axial lengths, ${ }^{7}$ which suggested that the myopic growth was not caused by increased eye temperature, nor by mechanical side-effects of the fused lids. Since during darkrearing neither eye became myopic, simple reduction in illumination was thought not to be a relevant cause. Rather, in light-rearing with lid-suture, the cause was thought to be a reduction in patterned retinal images, (together with $0.5 \mathrm{log}$. units of attenuation of light intensity).

Secondly, they reported in 1979 that opacification of the cornea by injection of latex particles produced an increase in axial length, ${ }^{8}$ measured by ultrasound, with no changes in the anterior segment of the eye. (Some corneal neovascularisation had occurred, and the latex had been ingested by macrophages.) This result can also be explained as the consequence of a reduction in patterned vision, but again rules out thermal or mechanical effects of lid-suture as primary causes of myopic overgrowth. Raviola and Wiesel pointed out a number of clinical analogies (discussed below) where poor image quality is associated with subsequent myopia.

Raviola and Wiesel $^{9}$ reported in 1985 an extension and overview of their work. In certain animals raised with lid suture a temporal crescent could be seen. They carried out further experiments to test for the role of the central nervous system on eye growth. Removal of the occipital cortex had no effect on lid-suture myopia. Atropine instillation had no effect on lid-suture myopia in three rhesus macaques, but prevented myopia in four stump-tailed macaques. (Although spasm of the ciliary muscle produced by isoflurophate did not enhance myopia in the stump-tailed macaque). Raviola and Wiesel also examined the effects of optic nerve section on lid-suture myopia. Here, in the stump tailed macaque (one animal) myopia was prevented by optic nerve section, while in the rhesus macaque (three animals) it was not, suggesting that in the rhesus monkey intraocular factors produce overgrowth: the retina releases 'regulatory molecules whose production is influenced by the pattern of light stimulation'. These rather complex experiments were described in their widely reviewed and influential paper "An animal model of myopia": it is clear that considerable work remains to clarify the apparent difference in response of the two species used - is this a sampling error or a real effect? It is also puzzling that in one species accommodation may have an effect on overgrowth, because of the dictum, which has not yet been refuted in man or monkeys, that accommodation is yoked on the two sides. If this is true, then unilateral deprivation should never produce myopisation of one eye only. A further query can be made on the dark rearing results. Darkrearing does not simply remove the visual input: it changes the circadian rhythms of the animal, and must modify numerous cyclical actions both systemically and within the eye. It has recently been reported by Guyton, Greene and Scholz ${ }^{10}$ that in the dark reared rhesus monkey, the eye remains close to the hypermetropia typical of its neonatal condition.

Raviola and Wiesel's results are very consistent in showing that lid-suture leads to myopic refraction. Other groups have not always obtained consistent results, for example von Noorden and Crawford, ${ }^{11}$ suggesting the possibility that emmetropisation is disrupted in either direction rather than growth being driven to myopia. Recently however, Smith, Hawerth, Crawford and von Noorden ${ }^{12}$ have examined results from their own studies and from other laboratories, where in a total of 43 out of 47 animals, uniocular suture leads to a more myopic refractive state. It should be noted that 'inconsistent' responses to lid suture have often been discussed in terms of variations in surgical technique, age, or other factors, but could actually be invaluable indicators of the type of system being disrupted (see 'Emmetropisation' below).

\section{Myopia and the avian eye}

There has been considerable interest in avian models of myopic growth, summarised by Yinon. ${ }^{13}$ In the newly hatched chick myopia can be produced rapidly as a consequence of lidsuture, and after wearing simple visual occluders. There are several advantages in studying the avian eye: vision in birds is a subject of wide neurobiological interest; birds 
are highly visual animals, making use of acute spatial vision (in the control of flight and in feeding), and are thus likely to be well-suited to examine the theory that image quality can regulate eye-growth. Furthermore, experiments on normal and experimentally modified eye growth can be carried out over a short timescale, in numbers sufficient to test a variety of mechanistic hypotheses, of neurobiological and ophthalmological relevance. Our studies ${ }^{14,15,16,17}$ have been of the pigeon (Columba livia) and the chick (Gallus domesticus).

\section{Lower field myopia in the pigeon eye}

In human vision, fine details of the visual world are gained only at the fovea. In a panoramic visual system such as that of the pigeon, detailed vision is provided over a wide expanse of visual space: cell densities remain high over considerable areas of the retina, ${ }^{18,19}$ and there is relatively little decline in acuity passing from the laterally directed optical axis to the frontal periphery. ${ }^{20}$ It is of considerable interest to compare the organisation of a panoramic and a foveocentric eye. When the pigeon is walking or feeding, it has to view the nearby ground and food, yet has to remain visually alert to distant predators and alarm signals.

We report electrophysiological evidence for a regional refractive adaptation in the visual field of the pigeon. Below the horizon the photoreceptors are near conjugate with the ground. At and above the horizon the eye is nearemmetropic. Nearby and distant objects (on the ground and above the horizon) are seen simultaneously in focus.

The literature contains persistent suggestions of sectorial variations in the refractive state of the pigeon's eye, ${ }^{21,22,23,24}$ with the frontal visual field myopic and the lateral visual field emmetropic or hypermetropic. Only the study of Erichsen ${ }^{24}$ has measured refractive state across wide angles in the visual field, with retinoscopy. However, off-axis retinoscopy is an extremely difficult technique. Further, the hypermetropic artefact of retinoscopy ${ }^{25}$ varies with eccentricity, and precludes absolute measurements of refractive state.

We used an alternative technique to refract the eye, which is objective, can provide absolute readings, and refracts the photoreceptor plane. We have recently developed a Maxwellian-view optometer, using Scheiner's principle, ${ }^{26,27}$ where defocus of a grating stimulus is accompanied by lateral image shift. A grating stimulus is carried in the field stop, and can be moved axially by a micrometer. The light sources, two light-emitting diodes are imaged in the pupil plane of the eye. The grating is imaged on the retina. When the grating is at the first principal focus of the final lens, it is conjugate with the photoreceptors if the eye is emmetropic. If the eye is myopic the grating becomes conjugate with the photoreceptors at a position closer to the final lens, and this distance from the first principal focus is proportional to refractive error. When the grating is out of focus at the photoreceptors, alternation between the light sources results in image shift on the retina. We detect the response to image shift as a small, focal, electroretinogram (ERG), after averaging. When the grating is conjugate with the photoreceptors, the ERG is minimal, ideally dropping to the level of residual noise. As defocus is introduced so the averaged responses rise on each side of this minimum. A refractive run is carried out by averaging the ERG with a range of optometer settings. The profile is fitted with a Vshaped function to determine the minimum point, which corresponds to the refractive state. Repeating such runs provides a measure of the accuracy of the refraction: 3-6 repetitions can result in SEMs of 0.2-0.4 D. In all experiments the beak-tip was positioned 35 deg below the pupil centre, since this head orientation is used in walking and in flight. ${ }^{28}$

The mean refractive state in the lateral field, on the horizon (azimuth $90 \mathrm{deg}$ ), was $+0.013 \mathrm{D}$, s.d. $0.97, n=76$. This is not significantly different from emmetropia, using Student's ttest. All refractive runs taken along the horizon were also essentially emmetropic. Vertical cuts through the visual field were made at azimuths 35,65 , and $90 \mathrm{deg}$, to sample the visual field symmetrically in front of and behind the optical axis. The results in each azimuth were markedly similar. Above the horizon all refractions are within $2 \mathrm{D}$ of emmetropia, and are well fitted by a straight line of zero slope. Thus the upper visual field is emmetropic. Below the horizon however there is a marked deviation from an "all-emmetropic" eye. Refractive states move to more myopic values at lower elevations.

Why should this be so? The effect is not due 
THE SINE MODEL

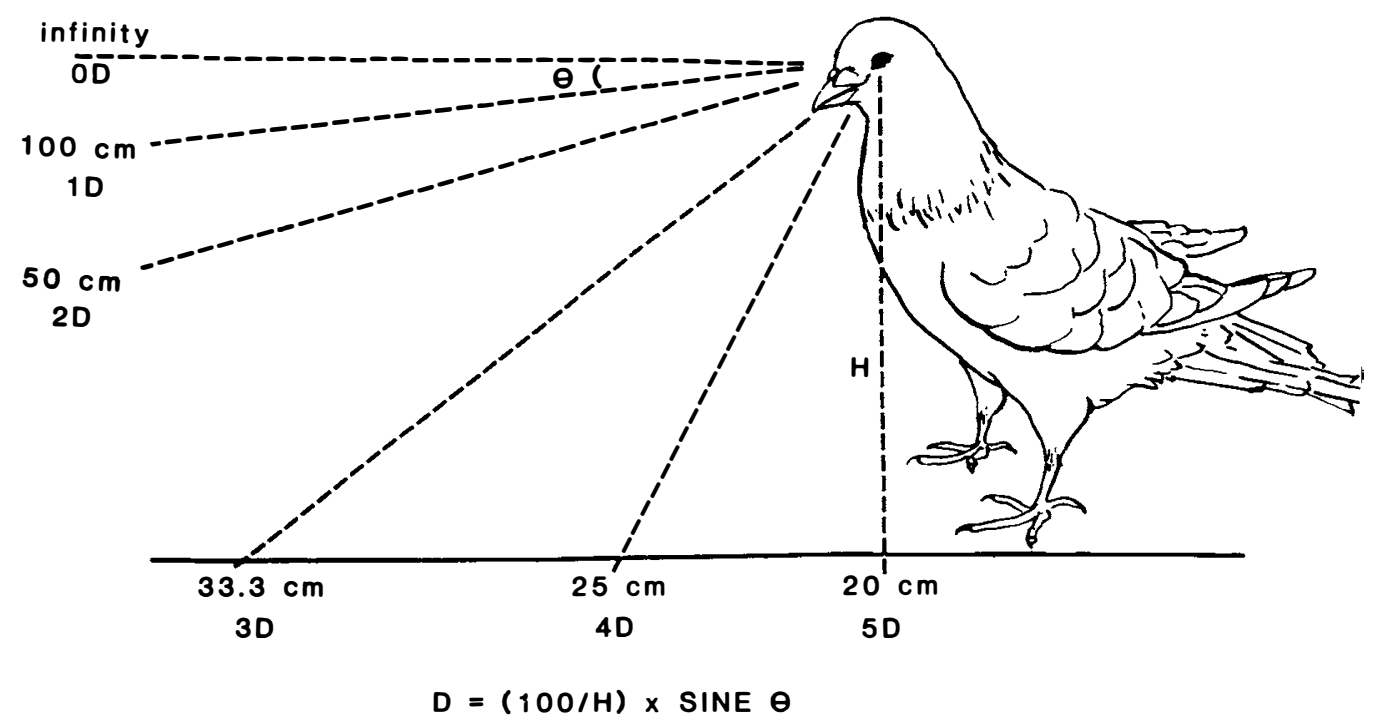

Fig. 1. A diagram showing how lower field myopia brings the entire ground-plane conjugate to the photoreceptors of the upper retina, when the pigeon is walking on the ground. A pigeon with eye-ground distance of $20 \mathrm{~cm}$ would need graded myopia following a sine-curve to attain 5D of myopia at 90 degrees below the horizon.

to astigmatism, which we have measured in control experiments, nor can it be due to any aberration of the eye symmetrical about the optical axis. We tested the idea that the eye is adapted to be focussed on the ground when the bird is walking. This would require lower field myopia to follow a sine curve, such that

$$
\mathrm{D}=(100 / \mathrm{H}) \cdot \operatorname{Sin} \theta
$$

Where D is the lower field myopia in Dioptres, $\mathrm{H}$ is the distance from eye to ground, and $\theta$ is elevation below the horizon. This sine model provides a maximal myopia at elevation $-90^{\circ}$, moving to emmetropia at the horizon. Sine curves were fitted to the data by computer, minimising the mean squared error for successive values of the parameter ' $H$ ', (the eye-toground distance). The best-fitting value of $\mathrm{H}$ was $20 \mathrm{~cm}$. We measured the eye-to-ground distance in 10 pigeons, and found it to be $18.6 \mathrm{~cm}$, s.d. $1.93 \mathrm{~cm}$. While the bird can adopt a range of raised or crouched postures, the agreement of eye-to-ground distance appears reasonable, and suggests that the photoreceptors in the upper retina are conjugate with the ground, for the frontal, axial, and lateral visual field.

Our findings show that there is a regional variation in refractive state in the pigeon eye, with a graded myopia in the lower visual field, and an emmetropic horizon and upper visual field. We find that the eye is emmetropic in frontal vision at and above the horizon: ie $35 \mathrm{deg}$ above the eye-beak axis. Since the pigeon flies and walks with its beak held $35 \mathrm{deg}$ down, ${ }^{28}$ forward vision is subserved by an emmetropic sector of the visual field. In frontal vision the eye must be emmetropic in the upper part of its binocular field, ${ }^{29,30}$ and myopic in the lower part. Figure (1) shows diagramatically how this lower field myopia brings the ground conjugate to the photoreceptors in the upper retina, when the bird is walking on the ground.

We do not know whether lower field myopia is unique to the eyes of granivorous birds. We suspect that it may be present in terrestrial animals which preserve optical quality and retinal 'grain' in peripheral vision. We speculate that a comparable organisation may exist in animals specialised for frontal vision, such as the cat or the burrowing owl. In these animals the 'vertical' horopter is at or close to the ground level, ${ }^{31}$ and the utility of precise binocular machinery might be enhanced if the photoreceptors of the upper retina were near-conjugate to the ground-plane. 
Many fascinating problems remain in the study of lower-field myopia in the pigeon, including a morphological and optical study of its basis. Is it the result of a well-controlled gene-plan, or is it locally regulated or fine-tuned during development of visual feedback? Does a comparable situation exist in the chick, which carries out foraging from its earliest posthatching days, over a period of time when eyeground height varies from a few centimetres to the adult state? Hodos and Erichsen ${ }^{32}$ have studied lower field myopia in a range of birds (day-old chicks, quail, pigeons and adult chickens) in which eye-ground height varies from $7 \mathrm{~cm}$ to $54 \mathrm{~cm}$. In these animals refractive state varies in satisfactory accord with the sine model bringing the ground conjugate to the upper retina. Hence in the chick, lower field myopia must adjust as the eye-ground height increases. There is therefore a regulation away from lower field myopia as growth proceeds.

\section{Lid-suture myopia in the chick}

The effects of lid-suture on the morphology and refractive state of the chick eye have been extensively described by Yinon and collaborators. ${ }^{33,34}$ The morphological changes included increased axial length, and increased equatorial diameter. In addition however there was a pronounced increase in depth of the anterior chamber, with an apparent bulging of the cornea. The net refraction, by retinoscopy, was $-21.9 \mathrm{D}$ for sutured eyes, in comparison to +0.9 D for open eyes. (These effects resemble those of full field occlusion, see below). Deprivation periods of 2-4 months were used. Thus far, the effects of refraction and on the posterior chamber, the chicks 'reared on a normal diurnal light/dark cycle, resemble those found in the monkey.

However, there has been an important series of experiments on eye growth in the chick in which the light/dark cycle has itself been varied, and these will be outlined before returning to the interaction of lid-suture and the light/dark cycle. It was reported in 1957 by Jensen and Matson ${ }^{35}$ that rearing in continuous illumination modifies eye-growth in the chick. Equatorial and axial dimensions are increased, the cornea is flattened, and the eyes develop reduced outflow facility and increased intra-ocular pressure. This fascinating model of avian glaucoma has been reviewed recently by Lauber. ${ }^{36}$ It has also been reported that dark-rearing results in enlargement of the posterior segment, accompanied by corneal flattening, a net hypermetropia, though not glaucoma. ${ }^{37,38}$ When lid-suture is combined with dark rearing, the cornea is flattened yet further, and hypermetropia is increased. Thus in the chick raised with lid-suture with a conventional light/dark cycle lid suture leads to myopic eye enlargement, increased anterior chamber depth and a bulging cornea. When lid-suture is combined with dark-rearing, the eye enlargement is accompanied by corneal flattening and hypermetropia. Different effects are generated in the anterior chamber and on refractive state by lid-suture, depending on the light/dark cycle.

Experimental myopia in the chick: occluders Recent interest in this myopia model has been stimulated by the important observations of Wallman, Turkel and Trachtman ${ }^{39}$ who reported in 1978 that high myopias, measured by retinoscopy, could be produced in the chick by restricting vision by simple occluders worn for 4-7 weeks after hatching. A high axial myopia was produced by occluding the whole visual field of one eye by a hemispherical dome, and by another device which permitted frontal vision only. A cylinder glued round the eye permitted lateral but not frontal vision: this device did not result in myopic refractions.

Our intention was to follow up the finding that the application of simple devices to the eye of the young chick can produce an increase in eyesize. ${ }^{40}$ We wished to establish whether these large eyes were indeed myopic, using a technique free from the small-eye artefact of retinoscopy, and to provide a morphological description of eye dimensions in normal and myopic eyes of sufficient precision for a ray tracing analysis. We hoped that the morphology of the anterior segment, including the outflow apparatus and the accommodatory system, might supply clues as to the cause of over-growth. Here we summarise the results of refractive studies and of eye morphology.

Three devices, domes, arches and rings, were applied to three-day old chicks. The domes degraded the image over the entire visual field of one eye. The arches degraded only the lateral visual field, leaving unobstructed vision in the frontal and posterior visual field. The rings did 
not occlude the visual field at all, but served as a control for possible mechanical impediments to growth of the other devices. Untreated chicks served as a fourth, control group.

We used devices as an alternative to lid-suture because they allow several further experimental designs to be carried out. The devices can carry ophthalmic lenses, and can be constructed so as to occlude only part of the visual field. It is of some interest to establish whether the sign or the magnitude of refractive error control the extent of induced myopia, and to know whether partial occlusion of the visual field (as with the arch device) results in myopisation of only part of the eyeball.

Chicks were refracted in the lateral visual field at ages of 3-7 weeks; if they had worn devices, they were refracted after the device was removed. Statistical analysis of the refractive states of the four groups was carried out by oneway analysis of variance, followed by Dunnett's test for comparing experimental group means to the mean of a control group. The results are summarised in Table (I) where $\mathrm{n}$ gives the number of refractions in each group:

The mean refractive states of normal and ring eyes did not differ significantly from each other, nor from emmetropia. Thus the application of the ring device produced no discernible effect on refractive state. The means of the arch and dome groups however differed significantly from each other, from emmetropia, and from the mean of the normal eyes $(p=<0.005)$. The dome device, in particular, produced a large shift to myopic refraction.

Morphological measurements were made from macrophotographs of the intact and hemisected eyes fixed as for electron microscopy. Effects of the devices upon eye dimensions were analysed from the mean differences between the treated (left) and untreated control (right) eyes, by analysis of variance, followed where appropriate by Tukey (a) tests and Student's ttests. The rings did not affect eye growth. The arch significantly increased the dorsoventral

Table I

\begin{tabular}{lcccc}
\hline & Normals & Rings & Arches & Domes \\
\hline mean ref raction & $-0.20 \mathrm{D}$ & $-0.19 \mathrm{D}$ & $-4.11 \mathrm{D}$ & $-14.88 \mathrm{D}$ \\
$\mathrm{s.d}$. & $1.3 \mathrm{D}$ & $0.88 \mathrm{D}$ & $3.0 \mathrm{D}$ & $8.03 \mathrm{D}$ \\
$\mathrm{n}$ & 45 & 32 & 33 & 30 \\
\hline
\end{tabular}

equatorial diameter of the eye. This effect suggests that myopia may be restricted to the sector of the retina undergoing visual deprivation: the view of the anterior and posterior retina was left unobstructed by the arch device.

The dome device had the most dramatic effects on eye morphology, and resulted in increases in both axial and equatorial dimensions. Dome eyes had a bulging cornea, increased anterior chamber depth, a more open angle, and a greater corneal diameter than control eyes. Axial length of the posterior segment was also increased. Figure 2 illustrates the morphology of a normal and a dome eye.

We have modelled an 'average' dome eye with ray-tracing. The main structural change is a $13 \%$ increase in the length of the posterior segment, which in itself introduces $18 \mathrm{D}$ of myopia. This effect is counteracted by the increase in anterior chamber depth, which reduces the net myopia to some $-15 \mathrm{D}$.

In our material we have noted no change in lenticular dimensions, nor in the accommodatory apparatus. Sivak and colleagues ${ }^{41,42}$ have reported that this experimental myopia has no effect on the size, protein content, or focal length of the chick lens. Thus as an optical organ the lens neither contributes to, nor compensates for, the induced ametropia.

In addition to the dimensional changes noted, several features were observed, particularly in dome eyes, which suggested that inflammatory responses may have occurred. There was an increased thickness of the choroid, and a cloudiness of the anterior vitreous, which extended from the ciliary body to the base of the pecten. These inflammatory reactions, and the swollen appearance of the circumorbital skin suggested that elevated temperature might play a role in the development of this form of experimental myopia. The role of temperature in the development of myopia in children following febrile illness has been of clinical interest. ${ }^{43}$ Elevated ocular temperatures during periods of eye growth, could encourage scleral growth, which could result in an elongated globe and a myopic refractive error.

In order to determine whether elevated eye temperatures occur during the development of experimental myopia, chicks were reared with domes, rings, or as untreated controls. ${ }^{44}$ At the end of the rearing period, they were anaesthe- 

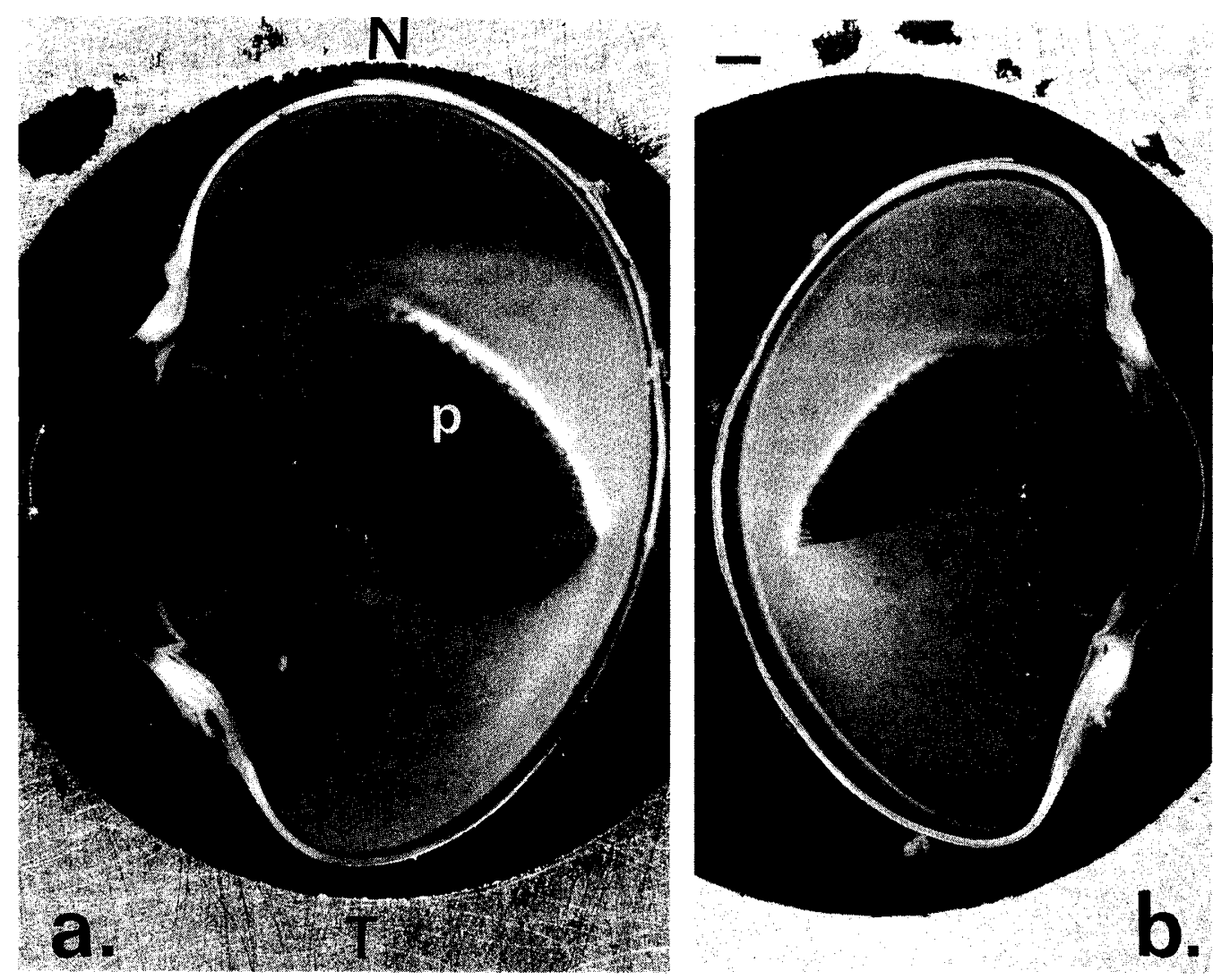

Fig. 2. Macrophotographs showing the ventral halves of the eyes of a chick aged 43 days. a) Left eye treated with a dome device for 40 days. The axial length of the dome eye is increased by $30 \%$ when compared with the untreated fellow eye (b). Equatorial diameter is greatest in the naso-temporal axis, increased by $13 \% \mathrm{~N}$ : nasal, T: temporal, $p$ : vascularised pecten (enlarged in the treated eye). Scale line $=$ Imm. Magnification = 4.5 times.

tised and a thermoprobe (a thermocouple in a 29 gauge needle) was inserted into the eye along the optic axis. Temperature readings were made at $1 \mathrm{~mm}$ intervals to a depth of $12 \mathrm{~mm}$. Temperature readings also were taken of the circumorbital skin and the air inside the dome. The results indicated that the temperature of the circumorbital skin was nearly $1^{\circ} \mathrm{C}$ warmer than the comparable contralateral tissue. The air temperature inside the dome, close to the cornea was nearly $4^{\circ} \mathrm{C}$ warmer than the air at the control cornea. The internal temperatures of the dome eyes were elevated from 2.8 to $5.2^{\circ} \mathrm{C}$ at the cornea, and 0.7 to $2.0^{\circ}$ at the axial sclera. Smaller elevations were found in the ring eyes. Two dome chicks that had lost their devices 24-28 hours prior to temperature measurement had thermal gradients that were nearly identical to those from untreated control eyes. The results suggest that the domes contribute to the development of this type of experimental myopia by trapping heat radiated from the eye. We have observed further that when chicks are raised in the dark with either domes, arches, crescents or rings, only the dome chicks develop an enlargement of the globe, which is equivalent to about one third of the enlargement of light-reared chicks.

Our experiments on the chick show that we have a convenient and reproducible model for producing large myopic shifts in a period shorter than two months. We are currently measuring intra-ocular pressure in normal and experimentally myopic chick eyes, for it is clearly 
important to test how IOP is involved in the eye enlargement. The morphological changes we observe in dome eyes are unilateral, and do not resemble the flattening of the cornea and reduction in anterior chamber depth found in avian glaucoma produced by rearing in continuous illumination. However, since clinical observations in man suggest that there may be some intersection of myopic and glaucomatous changes, it is reasonable to expect some analogous overlap in the chick, and dome produced myopia provides an ideal model to test this rigorously.

\section{Local myopias}

Our results with the arch device are compatible with myopisation being restricted to the visually deprived sectors of the retina. Wallman, Gottlieb, Rajaram and Fugate-Wentzek have pursued this idea, ${ }^{45}$ showing that wearing a device like a partial dome, with a removed sector giving clear vision to one part of the retina, gives an increased lens-retina distance only in the deprived portion of the retina. A 'local' myopia is produced. This local change in eye shape has intriguing analogies with the posterior staphyloma of progressive myopia. This argues for both effects that the cause of the myopia cannot be a general intraocular stimulus such as raised pressure, a raised temperature, or a secreted chemical which acts uniformly on the posterior pole. If there is such a general stimulus, it must be coupled with a localised susceptibility confined, in the experimental case, to the deprived region. This experiment cannot easily be performed on the monkey, because its large repertoire of eye-movements would reduce the effect of partial occlusion, and it is doubtful whether there would be sufficient behavioural compliance. It might however prove possible to implant an intraocular occluder to test whether local myopisation can occur.

In the chick this experimental local myopia persists after optic nerve section. ${ }^{46}$ Thus the overgrowth is produced by systems confined to the eyeball, and need involve no visual feedback from brain to eye (via accommodation or via the efferent system). A further result of this experiment is that nerve section itself resulted in an eye of reduced size, and of hypermetropic refraction.

An explanation could be that retinal cells sensitive to image quality can produce trophic substances which locally modify scleral growth or relaxation. It remains to be seen how such signals cross from neuroretina to sclera. It would seem unlikely that a freely diffusible substance could traverse the vascular bed of the choroid and yet produce local actions.

\section{Ophthalmic lenses and experimental myopia}

One of the problems with lid-suture or the use of occluders which are by design translucent or become so during use, is that the effects on the retinal image are both drastic and poorly defined. There is a loss of form vision, implying an extreme attenuation of all except a quasi-DC term in spatial frequency. There is a concomitant loss of the time-varying inputs derived from adequate imagery of high contrast, and reduction to the temporal frequency of a 24 hour cycle $(0.0000012 \mathrm{~Hz})$. If there is 'feedback' control of posterior chamber growth, with reduced contrast or imagery as an error signal, the signal generated by lid-suture can never be reduced as growth proceeds. (Perhaps this is why, even in the monkey, the effects are large).

It is for these reasons that lenses which introduce moderate defocus can lead to further crucial experiments on experimental myopia: they permit investigation of whether the eye can detect the sign and the magnitude of defocus, and use these signals to control compensatory growth.

The design of such experiments depends critically on the way in which accommodation is controlled, and the lenses are applied. If accommodation is yoked or symmetrical for the two eyes, (as in man or monkeys) and a lens is applied to one eye only, the animal will accommodate using the other eye, and the test eye will receive a blurred image, with sign and magnitude dependent on the lens used, and on the time-averaged accommodation generated by the 'control' eye. In the chick however, accommodation can take place independently in the two eyes, ${ }^{47}$ hence the two eyes could react independently to the ophthalmic lenses.

If the act of accommodation contributes to myopic development (a re-enactment of the 'close-work' connection), then negative lenses should have a greater effect than positive lenses. If the eye can accommodate, or relax accommodation, throughout the range of lenses used, then the amount of image blur will tend to be minimal for all lenses used. 
Negative lenses might be expected to have greater effects on eye growth than positive lenses even in the absence of accommodation. Consider a hypothetical growing eye which is emmetropic, and is rendered incapable of accommodation. A negative lens (say -4D) places the image shell behind the retina, conjugate to a physically unrealisable surface beyond infinity. Objects close to the eye are viewed with the greatest focus error, and objects at all distances from the eye are out of focus. If a positive lens of $4 \mathrm{D}$ is applied to the eye, the image shell is conjugate to a surface at $25 \mathrm{~cm}$ from the eye; this is both physically realisable and useful in visual behaviour. In other words, when the world is viewed through a positive lens, a surface in visual space will continue to provide wellfocussed images. (Exactly analogous arguments apply to the hypermetropic and myopic eye: a hyperetrope incapable of accommodation can see nothing in focus, whereas a myope is always conjugate to a close, well focussed surface in visual space.)

In applying lenses, it is important to ensure that they remain clean and clear, otherwise the lens will act as an occluder leading to profound loss of image quality. If lenses are mounted in a hood or helmet, it is important to check the extent to which this limits the visual field as an opaque occluder.

Experiments reported so far have used a single power of lens for the test eye. It would in principle be possible to replace lenses on a regular basis, with a gradual increase of power, so that compensatory growth, if occurring, could be driven to greater limits.

Schaeffel, Glasser and Howland ${ }^{48}$ fitted chicks with ophthalmic lenses $(-8 D$ to $+4 D$ in power). They showed by photoretinoscopy that the chicks could shift their non-cyclopleged refractive state to partially compensate for the lenses, although no relationship was demonstrated between lens power and refractive state either for positive or for negative lenses. Postmortem measurements of posterior nodal distance showed this to be greater for eyes wearing negative lenses than for eyes wearing positive lenses. (It is not clear from this paper that eyes wearing positive lenses differ from normal eyes in refraction or in posterior nodal distance. If the PND is smaller than in normal eyes, it would suggest that positive lenses retard axial growth.)
Schaeffel, Troilo, Wallman and Howland ${ }^{49}$ have repeated this experiment when accommodation is prevented by lesions of the Edinger-Westphal nucleus. Again eyes wearing negative lenses were more myopic than those treated with positive lenses, and of greater axial length. Hodos, Bessette, Wilkinson and Kuenzel ${ }^{50}$ have carried out experiments on the chick similar in design, but with lenses viewing the frontal visual field. No changes were found in axial dimensions (the lateral field was not defocussed by the lens), but nasotemporal equatorial diameter was increased, both by minus and plus lenses. This occurred in graded fashion as lens power increased, suggesting that magnitude but not sign of defocus drives the increase in size.

Experiments with lenses have been carried out in kittens by Smith, Maguire and Watson, ${ }^{51}$ in which negative lenses between -10 and $-16 \mathrm{D}$ resulted in increased axial length and myopia in the test eye. The control eye being viewed through a plano lens. Smith Harwerth and Crawford $^{52}$ used a similar method in four young rhesus monkeys. In two of them, a $-10 \mathrm{D}$ lens worn for 60-90 days resulted in substantial myopia, and increased axial length. The control eyes which had viewed through a plano lens were also myopic, to a slightly lesser degree. Conflicting results have been reported by Crewther, Nathan, Kiely, Brennan and Crewther, ${ }^{53}$ that young monkeys wearing extended-wear contact lenses of +6 to $-9 D$ became axially hypermetropic.

There are other manipulations to alter refractive state. One would be by refractive surgery, either by knife or by laser. Laser surgery could in principle sculpt a hypermetropic or myopic corneal surface, and it would be of interest to examine its stability during eye growth, and its effects upon axial length.

Another is to remove the lens. This has been done in neonatal monkeys by Wilson, Fernandes, Chandler, Tigges, Boothe and Gammon. ${ }^{54}$ The aphakic eyes were shorter in axial length than the fellow eyes, whether they had been optically corrected by contact lenses (11 eyes) or not (one eye). This experiment has the important clinical implication that lensectomy can result in undergrowth. This could remain a predominant effect even if aphakic eyes were used as models to test for the effect of hypermetropic blur on eye growth. 


\section{Recovery from experimental ametropia}

Wallmann and Adams ${ }^{55}$ investigated the susceptibility to and recovery from axial myopia in the chick produced by occluders which only permit frontal vision. Recovery can occur if the occluders are removed in the first six weeks, and seems dependent on a reduction in the growth rate of the posterior segment. The recoveries could be remarkably fast, and could amount to $20 \mathrm{D}$ in two weeks. This raises intriguing questions as to how an eye which can be 25D myopic in lateral vision (conjugate to a surface $4 \mathrm{~cm}$ from the eye, and defocussed at greater distances) can register this state correctly, and retard axial growth. In contrast, as described above, a chick eye viewing laterally through a $-2 \mathrm{D}$ or $-4 \mathrm{D}$ lens can initiate axial elongation.

Troilo and Wallman ${ }^{56}$ have reported that chick eyes made hypermetropic by dark rearing, or myopic by occluders, can recover towards emmetropia when reared in normal visual conditions. Recovery can proceed when the Edinger-Westphal nucleus is lesioned (eliminating accommodation) and when the optic nerve is cut. It is clearly important to know how anterior segment morphology adjusts in recovery, and to know whether the induced ametropias are inherently temporary effects, with 'recovery' being the result of resumption of normal eye growth (reduction in axial growth in myopic eyes, and recovery of anterior chamber depth in hypermetropic eyes).

\section{Pharmacological interventions}

There have been several studies of the effects of pharmacological agents on experimental myopia. As was mentioned above, Raviola and Wiesel $^{9}$ found that atropine had no effect in the rhesus macaque, but prevented axial elongation in the stump-tailed macaque.

A diet with increased glycine has been shown to reduce body growth yet result in macrophthalmos in the chick by Grosche, Anderson and Briggs. ${ }^{57}$ These macrophthalmic eyes have neither raised intraocular pressure nor reduced outflow facility. ${ }^{58}$

Wildsoet and Pettigrew have shown that the neurotoxin kainic acid has dose dependent effects on the growth of the chick eye. ${ }^{59}$ Twenty nmole injected intravitreally resulted in axial and equatorial enlargement, and a bulging cornea. A 200 nmole injection resulted in axial and equatorial enlargement, but corneal flattening. Thus the effects of the lower dose resemble those of lid suture or occlusion, while the higher dose produces effects akin to continuous illumination. If the effects on the posterior chamber are due to the reduction of retinal activity via neurotoxicity, there may be analogies with image impoverishment in lid-suture myopia. Reduced activity or reduced imagery would then be associated with an increased release of 'growth factor', or with a decreased release of 'growth inhibitor'. Kainic acid is a glutamate analog. Another glutamate analog, amino-phosphonobutyric acid (APB) has been reported to reduce axial growth and result in hypermetropia in the kitten eye by Smith, Fox and Duncan. ${ }^{60}$

Lin, Stone, Laties and Iuvone ${ }^{61}$ have observed that in retinae from chick eyes with lid-suture or occluders, the level of dopamine and its metabolite 3,4 dihydroxyphenylacetic acid is reduced. Stone, Laties, Raviola and Wiesel ${ }^{62}$ have reported that in rhesus eyes, lid-suture can result in an increase of vasoactive intestinal peptide (VIP) in the inner plexiform layer, as judged by immunohistochemistry. The effect occurred both in eyes of juveniles, which did become myopic, and in an adult eye, which did not. No changes were observed in the levels of substance-P. Studies of VIP levels in the retina, made for other purposes, suggest that lid-suture (together with facial nerve degeneration) can lead to a reduction in VIP in the rabbit retina, as reported by Butler, et al. ${ }^{63}$ Eriksen and Larsson $^{64}$ have reported that dark-rearing can result in a reduction of VIP immunoreactivity in the rat.

The eventual aim of these experiments will be to associate drug actions with specific cell or receptor types in the retina or in the uvea, given that direct actions on scleral growth can be ruled out, and to test whether identified changes are a cause rather than an effect or side-effect of myopic overgrowth. If, for example a particular modulator is thought to be involved in producing myopic overgrowth, can a specific antagonist block the overgrowth? There is evidence that neurotransmitters or modulators can influence morphogenesis of retinal neurones. ${ }^{65}$ They are well placed as potential carriers of signals from retina to sclera, although the messenger systems, and indeed the messages, remain to be discovered. 


\section{Clinical Models of experimental myopia}

A number of clinical observations suggest that there may be a condition of image deprivation myopia in man. Birth injuries of the cornea, often unilateral, have been associated with subsequent myopia, ${ }^{66}$ and in certain cases the myopia has been shown to be present in an eye of increased axial length ${ }^{67}$ Myopia and increased axial length have been found in patients with corneal opacities. ${ }^{68}$ Johnson, Post, Chalupa and Lee ${ }^{69}$ have described an axial myopia in one eye, which had a posterior subcapsular cataract, in one sibling of a pair of identical twins. Von Noorden and Lewis $^{70}$ examined 10 young patients who had unilateral cataracts removed: in seven out of 10 cases the involved eye was of increased axial length. These cases can be regarded as suggestive analogies to experimental corneal opacification.

Robb $^{71}$ has described an association between hemangiomas of the eye and orbit, and subsequent ametropia, often a high myopia, in young children. Hoyt, Stone, Fromer and Billson ${ }^{72}$ have described the association between neonatal lid closure, caused by nerve palsy, obstetric trauma, and hemangioma, and axial myopia in eight infants. These cases can be regarded as parallels to the lid-suture experiments.

Rabin, van Sluyters and Malach $^{73}$ have described the association between a variety of monocular visual anomalies, including retrolental fibroplasia, persistent pupillary membrane, vitreous debris, and myopia. They also showed an associated myopic error in a number of patients with binocular anomalies, including RLF and congenital cataract. They discuss their findings in terms of deprivation produced myopia resulting from interruption of the emmetropisation process.

\section{Emmetropisation}

The term 'emmetropisation' has been used in a variety of ways in ophthalmology. One is to describe the process by which neonatal refractive state (in man slightly hypermetropic) is both reduced in variance and shifted in mean value, so that as eye growth progresses, the mean refraction becomes near-emmetropic. A similar change in refractive state has been described in the chick. ${ }^{74,42}$ During eye growth in man the power of the eye declines from some $85 \mathrm{D}$ to $60 \mathrm{D}$, and all optical components participate in co-ordinated growth.

It has been supposed that this process is vision-dependent, and dependent on good image quality. A genetic program for eye growth could specify in outline the growth rates of the ocular components, with a superimposed rule 'If the image is blurred, increase axial length'. This rule would ensure growth from infant hypermetropia to adult emmetropia. If this rule operated in adulthood, and the eye had overshot to a myopic error then there would be an ineluctable progression to greater myopia, if a blurred image caused scleral relaxation. A rule as simple as this could explain emmetropisation, lid-suture myopia, myopia of occluders, the local myopias, the forms of image deprivation myopia in man, and by extension, progressive myopia. It could explain the low-grade 'physiological ametropias' (myopic and hypermetropic) as due to a greater or lesser susceptibility to the signals inducing axial growth. This rule would predict that both positive and negative lenses produce axial growth.

This rule for emmetropisation explains so much so easily that a high degree of scepticism should be retained towards it.

Biologically, it would be maladaptive to make eye growth vulnerable to poor imagery in all circumstances: for example in animals which are born with their eyes closed, and optically immature, with retention of embryonic vasculature (like the cat), or which spend an appreciable time asleep (like human infants), or when accommodation takes an appreciable time to develop post-natally (as in man). If in man the growing eyes were highly vulnerable to focus error, myopic progression should be easily halted by distance correction, corrected hypermetropic eyes would be smaller than uncorrected ones; and penalisation with atropine would be myopia-inducing. None of these expectations seems to be true. Further, in many circumstances the image shell must contain both focussed and defocussed images simultaneously, depending on the distance of objects in the visual world.

There is a further puzzle with an 'image starvation' theory of myopic growth, arising from the comparison of lid-suture and dark rearing, for both procedures result in excision of high contrast imagery, yet, in the monkey only one leads to axial myopia. If the differing effects are caused by other factors (mean level of illumination, differences in the light/dark cycle, and so on,) these factors require investigation. In the 
chick both procedures can result in increase in length of the posterior segment (with differing effects on the anterior chamber) so; arguably, the rule is followed.

A development of the simple theory outlined above would be that the growing eye can sense both image focus and the direction of defocus errors, and use the direction or sign of the error to speed up or slow down axial growth. (Growth of the anterior segment might also be supposed to be under collaborative control). This theory would be supported if it were found that negative lenses (and hypermetropia) increase axial growth, and that positive lenses (and myopia) retard axial growth. While some sub-set of these phenomena is suggested by the experimental myopias, further hard evidence is needed. If regulation of this sort applied to the human eye, one would expect reports of emmetropisation from an initially myopic state in childhood. Since myopia can progress, both in childhood eye growth and during adulthood, it must be argued that 'defocus and sign' regulation is not simply falling into random error, but persistently taking the wrong bifurcation, and producing axial elongation where retardation is required.

Let us consider, briefly, some logical consequences of a 'defocus and sign detection' rule for eye growth. It is well established that lidsuture and visual occlusion can lead to large amounts of myopic overgrowth. Why should a 'defocus and sign' regulator treat the abnormal visual input as a hypermetropic error? The image cannot be improved by accommodation, as could a hypermetropic focus error; and only a hypermetropic error should lead to compensatory axial growth. Indeed, myopic overgrowth caused by lid-suture can occur after optic nerve section.

Thus while a 'defocus and sign' rule can explain emmetropisation, it does not provide a clear explanation for lid-suture myopia, nor for the myopia of full-field occluders, nor for the local myopias, nor for the human myopias associated with image deprivation. This is because these conditions are not readily identifiable by a regulator seeking the sign of a refractive error. (Perhaps the problem of identifying the sign of a refractive error underlies the inconsistencies reported for the primate model.) If it is argued that the sign of the refractive error is signalled by preserved imagery for close objects for the myopic eye, then the theory becomes a version of the blur theory: different ametropias providing different combinations of defocus terms, with the most impoverished image being found in high hypermetropia and in lid-suture. The blur theory can be supported by the evolutionary argument that for a visual animal high hypermetropia is a more maladaptive refractive state than myopia, and suggests the paradoxical insight that the experimental myopia and deprivation myopias arise because retinal mechanisms believe themselves to be in a highly hypermetropic eyeball.

Thus the first rule we considered 'If the image is blurred, increase axial length' provides wider explanatory power than the second, based on 'Defocus and sign detection'. It also has the merit of being simpler for retinal circuitry to implement, merely by measuring the modulation depth of responses of neurones with spatially restricted receptive fields, to space-varying and time-varying stimuli. However, choice between these theories is now a matter for active experimentation. If different vertebrate classes follow different rules for eye growth, the evolutionary explanation will be of great fascination.

\section{Conclusions}

This review has given a brief account of animal experiments which provide a range of models for myopia growth, and show how myopia can be induced by simple environmental and optical factors. Myopia in the lower visual field can be demonstrated in some animals, where it can be of adaptive value in foraging and ground feeding.

An account has been given of clinical observations which provide parallels to the induced myopias, suggesting that 'image deprivation' in early life can lead to axial myopia.

The experimental analysis of myopic growth is both of biological and of ophthalmological interest. Many important experiments remain to be carried out. Would prolonged lid-suture lead to the posterior pole pathology of progressive myopia? At present, only a myopic crescent has been reported. A fuller clarification of the defocus conditions leading to myopia is needed. The neural circuitry responding to the altered visual input is not yet specified, and we only have hints as to its neuropharmacology. If signals 
are generated in the retina which ultimately and perhaps locally influence scleral growth, the cell systems carrying these messages remain unknown. Knowledge of the cell biology and molecular biology of these messages could provide the possibility of clinical intervention into myopic progression, and could provide important insights into chorioretinal and scleral disease.

We are grateful for financial support from: The Moorfields Eye Hospital, The National Eye Institute (USA), The Royal Society, and The Smith, Kline and French Foundation.

\section{References}

${ }^{1}$ Duke-Elder Sir W. Stewart. Text-book of Ophthalmology. Vol IV. London, Henry Kimpton. 1949.

${ }^{2}$ Duke-Elder Sir Stewart and Abrams D. System of Ophthalmology Vol V. London, Henry Kimpton. 1970.

${ }^{3}$ Blach Rolf K. Degenerative Myopia. In Krill AE and Archer DB Eds. Krill's Hereditary Retinal and Choroidal Diseases Vol II. Hagerstown, Harper and Row. 1977: 911-937.

${ }^{4}$ Curtin Brian J. The Myopias: Basic Science and Clinical Management. Philadelphia, Harper and Row. 1985.

${ }^{5}$ Wiesel TN, and Raviola E. Myopia and Eye enlargement after neonatal lid fusion in monkeys. Nature 1977; 266: 66-8.

${ }^{6}$ Heine L. Beitrage zur anatomie des myopischen Auges. Arch Augenheilk 1899; 38: 277-90.

${ }^{7}$ Raviola E, and Wiesel TN. Effect or dark-rearing on experimental myopia in monkeys. Invest Ophthalmol Vis Sci 1978; 17: 485-8.

${ }^{8}$ Wiesel TN and Raviola E. Increase in axial length of the macaque monkey eye after corneal opacification. Invest Ophthalmol Vis Sci 1979; 18: 1232-6.

${ }^{9}$ Raviola E, and Wiesel TN. An animal model of myopia. New Eng J Med 1985; 312: 1609-15.

${ }^{10}$ Guyton DL, Greene PR, Scholz RT. Dark-rearing interference with emmetropisation in the rhesus monkey. Invest Ophthalmol Vis Sci 1988; 29: (suppl) 33.

"von Noorden GK and Crawford MLJ. Lid-closure and refractive error in macaque monkeys. Nature 1978; 272: 53-4.

${ }^{12}$ Smith EL, Harwerth RS, Crawford MLJ, von Noorden GK. Observations on the effects of form deprivation on the refractive status of the monkey. Invest Ophthalmol Vis Sci 1987; 28: 1236-45.

${ }^{13}$ Yinon U. Myopia induction in animals following alteration of the visual input during development: a review. Curr Eye Res 1984; 3: 677-90.
${ }^{14}$ Fitzke FW, Hayes BP, Hodos W, Holden AL. Electrophysiological optometry using Scheiner's principle in the pigeon eye. $J$ Physiol $1985 ; 369$ : 17-31.

${ }^{15}$ Fitzke FW, Hayes BP, Hodos W, Holden AL, Low JC. Refractive sectors in the visual field of the pigeon eye. $J$ Physiol 1985; 369: 33-44.

${ }^{16}$ Hodos W, Fitzke FW, Hayes BP, Holden AL Experimental myopia in chicks: ocular refraction by electroretinography. Invest Ophthalmol Vis Sci 1985; 26: 1423-30.

${ }^{17}$ Hayes BP, Fitzke FW, Hodos W, Holden AL. A morphological analysis of experimental myopia in young chickens. Invest Ophthalmol Vis Sci 1986; 27: 981-91.

${ }^{18}$ Pumphrey RJ. Sensory Organs: Vision in Marshall AJ. (Ed) Biology and Comparative Physiology of Birds. New York. Academic Press: 1961.

${ }^{19}$ Hayes BP. The structural organisation of the pigeon retina. Progress in Retinal Research 1982; 1: 197-226.

${ }^{20}$ Ulrich DJ, Blough PM, Blough DS. The pigeon's distant visual acuity as a function of viewing angle. Vision Res 1982; 22: 429-31.

${ }^{21}$ Catania AC. On the visual acuity of the pigeon. $J$ Exp Anal Behav 1964; 7: 361-6.

${ }^{22}$ Millodot M, and Blough $\mathrm{P}$. The refractive state of the pigeon eye. Vision Res 1971; 11: 1019-22.

${ }^{23}$ Nye PW. On the functional differences between frontal and lateral visual fields of the pigeon. Vision Res 1973; 13: 559-74.

${ }^{24}$ Erichsen JT. 'How Birds look at Objects' D.Phil. Thesis, Oxford University, 1979.

${ }^{25}$ Glickstein M, and Millodot M. Retinoscopy and eye size. Science, $N Y$ 1970; 168: 605-6.

${ }^{26}$ Westheimer G. The Maxwellian View. Vision Res 1966; 6: 669-82.

${ }^{27}$ Emsley HH. Visual Optics. Vol 1, London, Hatton Press, 1952.

${ }^{28}$ Hodos W, Erichsen JT, Bessette BB, Phillips SJ. Head orientation in pigeons: postural, locomotor and visual determinants. Neurosci Abstr 1984; 10: 397.

${ }^{29}$ Martin GR, and Young SR. The retinal binocular field of the pigeon (Columba livia; English Racing Homer). Vision Res 1983; 23: 911-15.

${ }^{30}$ Martinoya G, Rey J, Bloch S. Limits of the pigeon's binocular field and direction for best binocular viewing. Vision Res 1981; 21: 1197-200.

${ }^{31}$ Cooper ML and Pettigrew JD. A neurophysiological determination of the vertical horopter in the cat and owl. J Comp Neurol 1979; 184: $1-26$. 
${ }^{32}$ Hodos W, and Erichsen JT. Lower-field myopia in birds: an adaptation that keeps the ground in focus. 1988 (personal communication).

${ }^{33}$ Yinon U, Rose L, Shapiro A. Myopia in the eye of developing chicks following monocular and binocular lid closure. Vision Res 1980; 20: 137-41.

${ }^{34}$ Yinon U, Koslowe KC, Lobel D, Landshman N, Barishak YR. Lid suture myopia in developing chicks: optical and structural considerations. Curr Eye Res 1983; 2: 877-82.

35 Jensen LS, and Matson WE. Enlargement of avian eye by subjecting chicks to continuous incandescent illumination. Science 1957; 125: 741.

${ }^{36}$ Lauber JK. Review: Light induced avian glaucoma as an animal model for human primary glaucoma. J Ocular Pharmacol 1987; 3: 77-100.

${ }^{37}$ Yinon U, and Koslowe KC. Hypermetropia in dark reared chicks and the effect of lid suture. Vision Res 1986; 26: 999-1005.

${ }^{38}$ Gottleib MD, Fugate-Wentzek LA, Wallman J. Different visual deprivations produce different ametropias and different eye shapes. Invest Ophthalmol Vis Sci 1987; 28: 1225-35.

${ }^{39}$ Wallman J, Turkel J, Trachtman J. Extreme myopia produced by modest change in early visual experience. Science 1978; 201: 1249-51.

${ }^{40}$ Hodos W, and Kuenzel WJ. Retinal image degradation produces ocular enlargement in chicks. Invest Ophthalmol Vis Sci 1984; 25: 652-9.

${ }^{41}$ Pickett-Seltner RL, Weerheim J, Sivak JG, Pasternak J. Experimentally induced myopia does not affect post-hatching development of the chick lens. Vision Res 1987; 27: 1779-82.

${ }^{42}$ Pickett-Seltner RL, Sivak JG, Pasternak JJ. Experimentally induced myopia in chicks: morphometric and biochemical analysis during the first 14 days after hatching. Vision Res 1988; 28: 323-8.

${ }^{43}$ Hirsch M. The relationship between measles and myopia. Am J Optom Arch Amer Acad Optom 1957; 34: 289.

${ }^{44}$ Hodos W, Revzin AM, and Kuenzel WJ. Thermal gradients in the chick eye: a contributing factor in experimental myopia. Invest Ophthalmol Vis Sci 1987; 28: 1859-66.

45 Wallman J, Gottleib MD, Rajaram V, FugateWentzek LA. Local retinal regions control local eye growth and myopia. Science $N Y$ 1987; 237: 73-7.

46 Troilo D, Gottleib MD, Wallman J. Visual deprivation causes myopia in chicks with optic nerve section. Curr Eye Res 1987; 6: 993-9.

${ }^{47}$ Schaeffel F, Howland HC, Farkas L. Natural accommodation in the growing chicken. Vision Res 1986; 26: 1977-93.
${ }^{48}$ Schaeffel F, Glasser A, Howland HC. Accommodation, refractive error and eye growth in chickens. Vision Res 1988; 28: 639-57.

${ }^{49}$ Schaeffel F, Troilo D, Wallman J, Howland HC. Chickens without accommodation become myopic or hyperopic from wearing lenses. Invest Ophthalmol Vis Sci 1988; 29: (suppl) 445.

${ }^{50}$ Hodos W, Bessette BB, Wilkinson JL, Kuenzel WJ. Frontal field lenses produce equatorial enlargement in chick eyes. Invest Ophthalmol Vis Sci 1988; 29: (suppl) 33.

51 Smith EL, Maguire GW, and Watson JT. Axial lengths and refractive errors in kittens reared with optically induced anisometria. Invest Ophthalmol Vis Sci 1980; 19: 1250-5.

52 Smith EL, Harwerth RS, Crawford MLJ. Spatial contrast sensitivity deficits in monkeys produced by optically induced anisometria. Invest Ophthalmol Vis Sci 1985; 26: 330-42.

${ }^{53}$ Crewther SG, Nathan J, Kiely PM, Brennan N, Crewther DP. Axial hypermetropia induced in primates. Invest Ophthalmol Vis Sci 1986; 27: (suppl) 202.

${ }^{54}$ Wilson JR, Fernandes A, Chandler CV, Tigges M, Boothe RG, Gammon JA. Abnormal development of the axial length of aphakic monkey eyes. Invest Ophthalmol Vis Sci 1987; 28: 2096-99.

55 Wallman J, and Adams JI. Developmental aspects of experimental myopia in chicks: susceptibility, recovery and relation to emmetropization. Vision Res 1987; 27: 1139-63.

${ }^{56}$ Troilo D, and Wallman J. Experimental emmetropization in chicks. Invest Ophthalmol Vis Sci 1988; 29: (suppl) 76.

${ }^{57}$ Groschke AC, Anderson JO, Briggs GM. Peculiar enlargement of eyeballs in chicks caused by feeding a high level of glycine. Proc Soc Exp Biol Med 1948; 69: 488.

${ }^{58}$ Helmsen RJ, Gaasterland DE, Rubin M. Induction of buphthalmos in chicks fed an excess of glycine. Invest Ophthalmol 1973; 12: 348-53.

${ }^{59}$ Wildsoet CF, and Pettigrew JD. Kainic acid induced eye enlargement in chickens: differential effects on anterior and posterior segments. Invest Ophthalmol Vis Sci 1988; 29: 311-19.

${ }^{60}$ Smith EL, Fox DA, Duncan GC. Refractive error changes in kitten eyes produced by chronic onchannel blockade. Invest Ophthalmol Vis Sci 1985; 26: (suppl) 331.

${ }^{61}$ Lin T, Stone RA, Laties AM, Iuvone PM. Altered dopamine metabolism and form deprivation myopia. Invest Ophthalmol Vis Sci 1988; 29: (suppl) 33.

62 Stone RA, Laties AM, Raviola E, Wiesel T. Increase in retinal vasoactive intestinal polypeptide after eyelid fusion in primates. Proc Natl Acad Sci 1988; 85: 257-60. 
${ }^{63}$ Butler JM, Ruskell GL, Cole DF, Unger WG, Zhang SQ, Blank MA, McGregor GP, Bloom SR. Effects of VIIth (facial) nerve degeneration on vasoactive intestinal polypeptide and substance $P$ levels in ocular and orbital tissues of the rabbit. Exp Eye Res 1984; 39: 523-32.

${ }^{64}$ Eriksen EF, and Larsson LI. Neuropeptides in the retina: evidence for differential topical localisation. Peptides 1981; 2: 153-7.

${ }^{65}$ Lankford KL, DeMello FG, and Klein WL. $\mathrm{D}_{1}$-type dopamine receptors inhibit growth cone motility in cultured retina neurons: Evidence that neurotransmitters act as morphogenetic growth regulators in the developing central nervous system. Proc Natl Acad Sci 1988; 85: 2839-43.

${ }^{66}$ Lloyd RIL. Birth injuries of the cornea and allied conditions. Am J Ophthalmol 1938; 21: 359-64.

${ }^{67}$ Angell K, Robb RM, Berson FG. Visual prognosis in patients with ruptures in Descemet's membrane due to forceps injuries. Arch Ophthalmol 1981; 99: 2137-9.

${ }^{68}$ Muramatsu T, Sato A, Tokoro T. Influence of corneal opacities on development of myopia. Folia Ophthal Jap 1982; 33: 1084-8.
69 Johnson CA, Post RB, Chalupa LM, Lee TJ. Monocular deprivation in humans: a study of identical twins. Invest Ophthalmol Vis Sci 1982; 23: $135-8$.

${ }^{70}$ von Noorden GK, and Lewis RA. Ocular axial length in unilateral congenital cataracts and blepharoptosis. Invest Ophthalmol Vis Sci 1987; 28: 750-2.

${ }^{71}$ Robb RM. Refractive errors associated with hemangiomas of the eyelids and orbit in infancy. Am J Ophthalmol 1977; 83: 52-8.

${ }^{72}$ Hoyt CS, Stone RD, Fromer C, Billson FA. Monocular axial myopia associated with neonatal eyelid closure in human infants. $A m \quad J$ Ophthalmol 1981; 91: 197-200.

${ }^{73}$ Rabin J, Van Sluyters RC, Malach R. Emmetropisation: a vision dependent phenomenon. Invest Ophthalmol Vis Sci 1981; 20: 561-4.

74 Wallman J, Adams JI, Trachtman JN. The eyes of young chickens grow toward emmetropia. Invest Ophthalmol Vis Sci 1981; 20: 557-61. 